\title{
IMAGING THE SMALL-SCALE CIRCUMSTELLAR GAS AROUND T TAURI STARS
}

\author{
D. W. KOERNER \\ Jet Propulsion Laboratory, 169-506, 4800 Oak Grove Drive, Pasadena, California 91109 \\ Electronic mail: davidk@coma.jpl.nasa.gov \\ A. I. SARGENT \\ Division of Physics, Mathematics and Astronomy, 105-24, California Institute of Technology, Pasadena, California 91125 \\ Electronic mail: afs@astro.caltech.edu \\ Received 1995 January 20; accepted 1995 January 25
}

\begin{abstract}
We have detected circumstellar molecular gas around a small sample of $\mathrm{T}$ Tauri stars through aperture synthesis imaging of $\mathrm{CO}(2 \rightarrow 1)$ emission at $\sim 2^{\prime \prime}-3^{\prime \prime}$ resolution. RY Tauri, DL Tauri, DO Tauri, and AS 209 show resolved and elongated gaseous emission. For RY Tau, the deconvolved, half-maximum radius along the direction of elongation, P.A. $\sim 48^{\circ}$, is $110 \mathrm{AU}$. Corresponding radii and orientations for the other sources are: DL Tau-250 AU at P.A. $\sim 84^{\circ}$; DO Tau $-350 \mathrm{AU}$ at P.A. $\sim 160^{\circ}$; AS $209-290 \mathrm{AU}$ at P.A. $\sim 138^{\circ}$. RY Tau, DL Tau, and AS 209 show velocity gradients parallel to the elongation, suggesting that the circumstellar material is rotating. RY Tau and AS 209 also exhibit double-peaked spectra characteristic of a rotating disk. Line emission from DO Tau is dominated by high-velocity blueshifted gas which complicates the interpretation. Nevertheless, there is in each case sufficient evidence to speculate that the circumstellar emission may arise from a protoplanetary disk similar to that from which our solar system formed.
\end{abstract}

\section{INTRODUCTION}

There is increasing evidence that the nebular environment assumed to have given rise to Solar System bodies is a common feature of the early stages of stellar evolution. Circumstellar dust disks are detected around a large fraction of T Tauri stars (e.g., Cohen et al. 1989; Strom et al. 1989; Beckwith et al. 1990; André \& Montmerle 1994; Henning et al. 1994) and in at least one case, the velocity field of the associated circumstellar gas is consistent with that of a model of a disk in Keplerian rotation (Koerner et al. 1993a, hereafter referred to as KSB). Disk properties, such as mass, size, and kinematics, are sufficiently similar to those expected for the early solar nebula (cf. Beckwith \& Sargent 1993a) that continued study is likely to afford us the opportunity to constrain theories of planetary system formation. A better understanding of the detailed properties of these circumstellar environments may also narrow down the field of truly protoplanetary disks.

The small fraction of T Tauri stars for which circumstellar gas has been observed (cf. Sargent \& Beckwith 1993) contrasts markedly with the $50 \%$ detection rate achieved by continuum surveys. This is largely because single telescope observations of the circumstellar dust continuum emission are readily obtainable. In general, unambiguous detection of circumstellar gas on small spatial scales requires molecular line millimeter-wave interferometer measurements; historically, these have been time consuming. Flattened structures of circumstellar gas with radii of order 1000 AU have been observed around the T Tauri stars, HL Tauri (Beckwith et al. 1986; Sargent \& Beckwith 1987), T Tauri (Weintraub et al. 1987, 1989); DG Tauri (Sargent \& Beckwith 1989; Ohashi et al. 1991), GG Tauri (Kawabe et al. 1993; Koerner et al. 1993b; Dutrey et al. 1994), and GM Aurigae (KSB). The earliest interpretations suggested that circumstellar material was centrifugally supported out to radial distances of 1000 AU (Sargent \& Beckwith 1987; Weintraub et al. 1989). Recent observations indicate that gas at these large radii is still in a state of collapse (e.g., Hayashi et al. 1993; van Langevelde et al. 1995). However, it also appears that some of these objects may not represent most T Tauri stars in general. For example, Hubble Space Telescope observations imply that HL Tau is heavily embedded in its parent cloud and is therefore at a very early stage of pre-main-sequence evolution (Stapelfeldt et al. 1995); the spectral energy distribution (SED) of T Tau, a binary object, can be understood only if there is a large component of nonplanar dust (Kenyon et al. 1993), and GG Tau is part of an unusual quadruple star system and surrounded by a large torus (Leinert et al. 1991; Ghez et al. 1993; Koerner et al. 1993b; Dutrey et al. 1994).

To date, GM Aur seems the best example of a "typical" $T$ Tauri star. It is relatively unobscured with $A_{V}=0.1$ (Strom et al. 1989) and is significantly older than the other stars which show circumstellar gas. It is also not a binary and affords a closer example of the early Solar System environment than the large fraction of $\mathrm{T}$ Tauri stars with a stellar companion (Ghez et al. 1993). Here, there is unambiguous evidence that the surrounding molecular gas is centrifugally supported out to $1000 \mathrm{AU}$, but emission beyond a $150 \mathrm{AU}$ radius is tenuous compared to the other circumstellar structures observed. This star/disk system may well represent a planet-forming environment (KSB). Obviously, we would like to observe a range of circumstellar gaseous environments for a larger sample of $\mathrm{T}$ Tauri stars to determine detailed properties of the disks and generalize these to compare with the conditions in the early solar nebula. We are continu- 
TABLE 1. Star properties.

\begin{tabular}{ccccccc}
\hline \hline (1) & $(2)$ & $(3)$ & $(4)$ & $(5)$ & $(6)$ & $(7)$ \\
Source & $\alpha$ & $\delta$ & $F_{1.3 m m}$ & $\alpha_{I R}$ & $M_{*}$ & Age \\
& $(1950)$ & $(1950)$ & $(\mathrm{mJy})$ & $(2.2-12 \mu \mathrm{m})$ & $\left(M_{\odot}\right)$ & $\left(\times 10^{6} \mathrm{yr}\right)$ \\
\hline RY Tauri & $04: 18: 50.80$ & +281935.0 & $229^{a}$ & 0.05 & $1.69^{a}$ & $0.21^{a}$ \\
DL Tauri & $04: 30: 36.02$ & +251424.0 & $230^{a}$ & -0.37 & $0.56^{a}$ & $1.20^{a}$ \\
DO Tauri & $04: 35: 24.18$ & +260455.2 & $136^{a}$ & -0.33 & $0.72^{a}$ & $0.60^{a}$ \\
AS 209 & $16: 46: 25.24$ & -141656.5 & $300^{b}$ & -0.60 & - & - \\
\hline \hline
\end{tabular}

a) Beckwith et al. (1990)

b) André \& Montmerle (1994)

ing a program of aperture synthesis imaging of the environments of T Tauri stars with a view to building up a statistical base of information on the circumstellar gas. High resolution molecular line images of four more systems, RY Tauri, DL Tauri, DO Tauri, and AS 209 are presented and discussed here.

\section{Observations}

RY Tau, DL Tau, DO Tau, and AS 209 are among the strongest sources of millimeter continuum emission in the nearest star-forming regions (Beckwith et al. 1990; André \& Montmerle 1994). Their coordinates (Herbig \& Bell 1988) and relevant properties are listed in Table 1 . The spectral index, $\alpha_{2.2-12 \mu}$, was derived from $K$-band magnitudes (Cohen \& Kuhi 1979; Ghez 1993) and IRAS $12 \mu \mathrm{m}$ fluxes (Weaver \& Jones 1992). The Taurus sources are located at distances of 140 pc and AS 209, near the Ophiuchi dark cloud, is at 160 pc (Elias 1978; Whittet 1974; de Geus et al. 1990).

Observations of RY Tau, DO Tau, DL Tau, and AS 209 in the $1.3 \mathrm{~mm}$ continuum and $\mathrm{CO}(2 \rightarrow 1)$ line at $230.5 \mathrm{GHz}$ were made between 1993 November and 1994 March, using the five-element Owens Valley millimeter-wave array. Baselines up to $60 \mathrm{~m} \mathrm{E}-\mathrm{W}$ and $60 \mathrm{~m} \mathrm{~N}-\mathrm{S}$ provided an approximately circular $3^{\prime \prime}$ beam (FWHM) with area $\Omega_{B}=6.9 \times 10^{-10}$ sr for DO Tau and $\Omega_{B}=6.1 \times 10^{-10}$ sr for AS 209. More extended baselines, up to $100 \mathrm{~m} \mathrm{E}-\mathrm{W}$ and $\mathrm{N}-\mathrm{S}$, yielded spatial resolution of up to 2 " for RY Tau and DL Tau with $\Omega_{B}=3.6 \times 10^{-10} \mathrm{sr}$ and $\Omega_{B}=6.0 \times 10^{-10} \mathrm{sr}$, respectively. Cryogenically cooled SIS receivers on each telescope produced overall system temperatures of $500-1000 \mathrm{~K}$. The digital correlator was configured to have two bands of Hanning smoothed channels, $32 \times 1 \mathrm{MHz}$ and $96 \times 83 \mathrm{kHz}$, yielding spectral resolutions 1.30 and $0.11 \mathrm{~km} \mathrm{~s}^{-1}$, respectively. Each band was centered at $V_{\mathrm{LSR}} \sim 6.0-7.5 \mathrm{~km} \mathrm{~s}^{-1}$ for the various Taurus sources (cf. Hartmann et al. 1986) and $V_{\mathrm{HEL}}=-4.0$ $\mathrm{km} \mathrm{s}^{-1}$ for AS 209. Continuum measurements were made simultaneously in a broadband channel of width $1 \mathrm{GHz}$. The quasar, $0528+134$, was observed at 20 min intervals to calibrate visibility phases for DO, DL, and RY Tau; NRAO 530 was used for AS 209. The absolute flux density scale relied on measurements of Uranus and 3C 273. Maps made with the NRAO AIPS software package are centered on the posi-

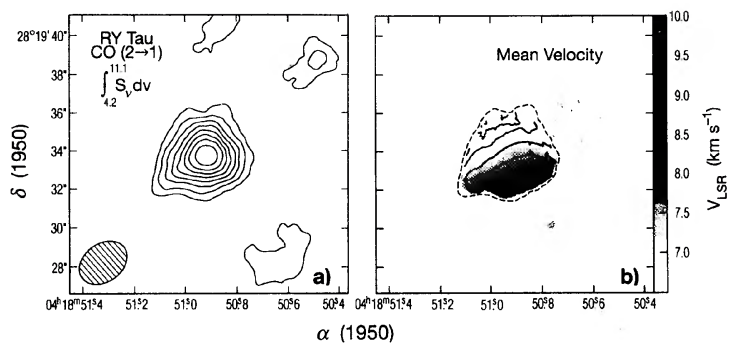

FIG. 1. (a) A map of $\mathrm{CO}(2 \rightarrow 1)$ emission toward RY Tau integrated over the velocity range, $V_{\mathrm{LSR}}=4.2-11.1 \mathrm{~km} \mathrm{~s}^{-1}$. The contour interval is 400 mJy beam ${ }^{-1} \mathrm{~km} \mathrm{~s}^{-1}(1 \sigma)$. Contours start at the $2 \sigma$ level. Peak emission is $3.9 \mathrm{Jy}_{\text {beam }}^{-1} \mathrm{~km} \mathrm{~s}^{-1}$ with an integrated intensity of $7.3 \mathrm{Jy} \mathrm{beam}^{-1} \mathrm{~km} \mathrm{~s}^{-1}$. The synthesized beam, 2. $6 \times 1.9$ FWHM at P.A. $-54.27^{\circ}$ with area $\Omega_{B}=3.6 \times 10^{-10} \mathrm{sr}$, is displayed as a hatched region. The apparent elongation to the SE is largely a beam effect. The FWHM deconvolved size of a Gaussian fit to the emission is $2.3 \times 1.9$ oriented at P.A. $48.6^{\circ}$, but this value is highly uncertain as an indicator of source size and orientation, since it is of order of the beam size. (b) A map of the mean velocity variations across the structure seen in (a). Greyscale values correspond to the velocity scale shown to the right. The contour interval is $0.5 \mathrm{~km} \mathrm{~s}^{-1}$. A systematic velocity gradient is evident along the axis of elongation. At the position of peak emission, the gradient is directed along P.A. $\sim 21^{\circ}$.

tions in Table 1. Uncertainties in absolute fluxes and positions are estimated to be $15 \%$ and 0.5 , respectively, for peak map intensities, but may be as high as $50 \%$ and one beamwidth $\left(\sim 2^{\prime \prime}-3^{\prime \prime}\right)$ for features detected at $3-5 \sigma$ levels.

\section{RESULTS}

Maps of the integrated $\mathrm{CO}(2 \rightarrow 1)$ emission for each source are displayed in Figs. 1(a)-4(a). Values for the integrated intensity are given in column (2) of Table 2 . For RY Tau and DL Tau, integration was over the velocity range for which emission appeared above a level of $300 \mathrm{mJy} \mathrm{beam}^{-1}$ $(2 \sigma)$ at spectral resolution $0.45 \mathrm{~km} \mathrm{~s}^{-1}$. Since the linewidths for AS 209 and DO Tau exceeded the velocity range of the high-velocity-resolution band, emission was integrated over the wider $1.30 \mathrm{~km} \mathrm{~s}^{-1}$ channels in which peak map intensity

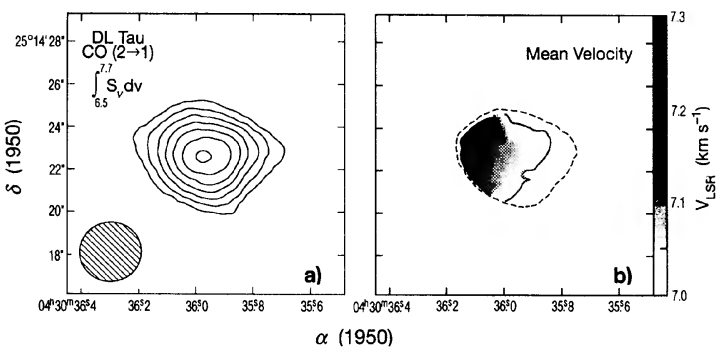

Fig. 2. (a) A map of $\mathrm{CO}(2 \rightarrow 1)$ emission toward DL Tau integrated over the velocity range, $V_{\mathrm{LSR}}=6.5-7.7 \mathrm{~km} \mathrm{~s}^{-1}$. The contour interval is 73 mJy beam ${ }^{-1} \mathrm{~km} \mathrm{~s}^{-1}(1 \sigma)$. Contours start at the $2 \sigma$ level. Peak emission is $549 \mathrm{mJy}^{-1} \mathrm{~km} \mathrm{~s}^{-1}$ with an integrated intensity of $1.1 \mathrm{Jy} \mathrm{beam}^{-1}$ $\mathrm{km} \mathrm{s}^{-1}$. The synthesized beam, $2.9 \times 2$ ". 8 at P.A. $-85^{\circ}$ with area $\Omega_{B}=6.0 \times 10^{-10} \mathrm{sr}$, is displayed as a hatched region. The FWHM deconvolved size of a Gaussian fit to the emission is $3.5 \times 2.5$ oriented at P.A. $84^{\circ}$, (b) a map of the mean velocity variations across the structure seen in Fig. 2(a). Greyscale values correspond to the velocity scale at the top. The contour interval is $0.1 \mathrm{~km} \mathrm{~s}^{-1}$. A gradient is evident along the axis of elongation at P.A. $\sim 85^{\circ}$. 


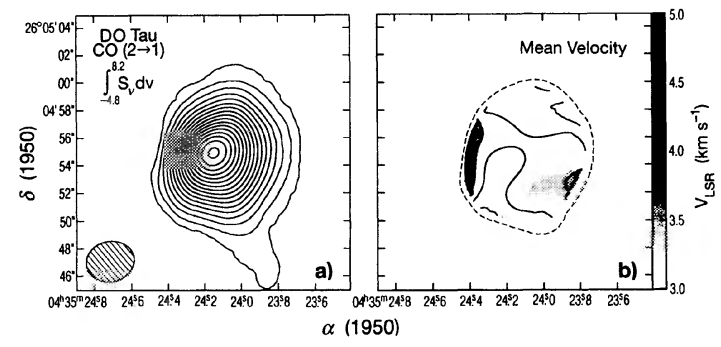

Fig. 3. (a) A map of $\mathrm{CO}(2 \rightarrow 1)$ emission toward DO Tau integrated over the velocity range, $V_{\mathrm{LSR}}=-4.8-8.2 \mathrm{~km} \mathrm{~s}^{-1}$. The contour interval is 2.0 Jy beam ${ }^{-1} \mathrm{~s}^{-1}(2 \sigma)$. Contours start at the $2 \sigma$ level. Peak emission is 34.5 Jy beam ${ }^{-1} \mathrm{~km} \mathrm{~s}^{-1}$ with an integrated intensity of $100.2 \mathrm{Jy} \mathrm{beam}^{-1} \mathrm{~s}^{-1}$. The synthesized beam, 3." $2 \times 2.9$ FWHM at P.A. $-54.27^{\circ}$ with area $\Omega_{B}=6.9 \times 10^{-10} \mathrm{sr}$, is displayed as a hatched region. The FWHM deconvolved size of a Gaussian fit to the emission is 5.1×3.9 oriented at P.A. $160^{\circ}$. The aspect ratio of the major and minor axes implies an inclination angle of $40^{\circ}$ for a circularly symmetric disk. (b) A map of the mean velocity variations across the structure seen in (a). Greyscale values correspond to the velocity scale shown to the right. The contour interval is $0.5 \mathrm{~km} \mathrm{~s}^{-1}$. A systematic velocity gradient is not easily identified. This may reflect a combination of kinematic effects, such as infall, rotation, and outflow.

was above a level of $50 \mathrm{mJy}^{\text {beam }}{ }^{-1}(2 \sigma)$. Individual velocity ranges are listed in column (3) of Table 2.

\subsection{Morphology, Optical Depth, and Mass}

To obtain the first approximate determination of the radial extent of the circumstellar gas, elliptical Gaussians were fit to the images shown in Figs. 1(a)-4(a) and deconvolved with the restoring beams from the CLEAN deconvolution process. The radius at half-maximum intensity, $R_{\mathrm{HM}}$, in column (4) of Table 2 is the FWHM major axis of this Gaussian fit. If the expected power-law profiles in temperature and density prevail, emission from the outer parts of disks is drastically reduced compared to the central regions. Thus, $R_{\mathrm{HM}}$ is a lower limit to the full radial extent of the gas. All of the

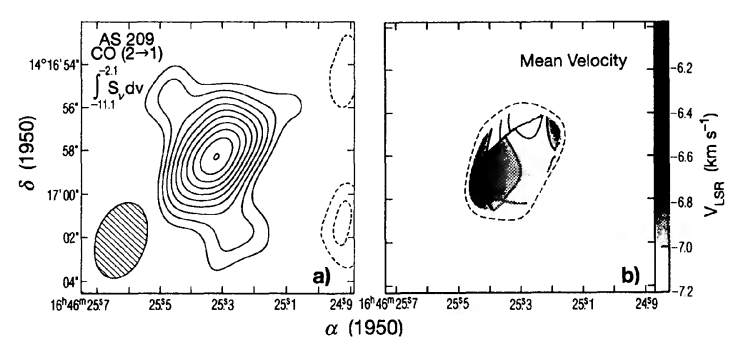

FIG. 4. (a) A map of $\mathrm{CO}(2 \rightarrow 1)$ emission toward AS 209 integrated over the velocity range, $V_{\text {hel }}=-11.1$ to $-2.1 \mathrm{~km} \mathrm{~s}^{-1}$. The contour interval is 350 mJy beam ${ }^{-1} \mathrm{~km} \mathrm{~s}^{-1}, 1 \sigma$. Contours start at the $2 \sigma$ level. Peak emission is 3.9 $\mathrm{Jy} \mathrm{beam}^{-1} \mathrm{~km} \mathrm{~s}^{-1}$ with an integrated intensity of $7.4 \mathrm{Jy} \mathrm{beam}^{-1} \mathrm{~km} \mathrm{~s}^{-1}$. The synthesized beam, 3."6×2.3 FWHM at P.A. $-18.6^{\circ}$ with area $\Omega_{B}=6.1 \times 10^{-10} \mathrm{sr}$, is displayed as a hatched region. The FWHM deconvolved size of a Gaussian fit to the emission is 3 ." $1 \times 2$ ".2 oriented at P.A. $137.9^{\circ}$. Low level contours are also elongated nearly perpendicular to this axis, as might be expected for $\mathrm{CO}$ entrained in an outflow. (b) A map of the mean velocity variations across the structure seen in (a). Greyscale values correspond to the velocity scale shown to the right. The contour interval is $0.25 \mathrm{~km} \mathrm{~s}^{-1}$. There is some indication of a velocity gradient along the $\mathrm{CO}$ elongation axis. Deviations from this trend may be due to outflow or infall components.
TABLE 2. Global properties derived from $\mathrm{CO}$ emission.

\begin{tabular}{cccccc}
\hline \hline (1) & $(2)$ & $(3)$ & $(4)$ & $(5)$ & $(6)$ \\
Source & $\int S_{\nu} d v$ & $\Delta \mathrm{V}$ & $\mathrm{R}_{H M}$ & $R_{\tau>1}$ & $M_{H_{2}}$ \\
& $(\mathrm{Jy} \mathrm{km} / \mathrm{s})$ & $(\mathrm{km} / \mathrm{s})$ & $(\mathrm{AU})$ & $(\mathrm{AU})$ & $\left(\times 10^{-6} M_{\odot}\right)$ \\
\hline RY Tauri & 6.6 & 6.9 & 107 & 52 & 9.6 \\
DL Tauri & 0.9 & 1.2 & 250 & 53 & 1.3 \\
DO Tauri & 100.2 & 13.6 & 350 & 164 & 145 \\
AS 209 & 7.4 & 10.4 & 287 & 115 & 10.7 \\
\hline
\end{tabular}

sources appear to be resolved in $\sim 2^{\prime \prime}-3^{\prime \prime}$ beams, with 100 $\mathrm{AU} \leq R_{\mathrm{HM}} \leq 400 \mathrm{AU}$. These dimensions are closer to those anticipated for the early solar nebula and approximately an order of magnitude lower than measured for other $\mathrm{T}$ Tauri stars.

As shown in KSB, an upper limit for the size of a central optically thick region in a circumstellar disk can be derived from the brightness temperature of peak emission at the central velocity. Assuming a typical disk temperature of $T_{k}=30$ K (Beckwith \& Sargent 1993b), an inclination angle of $45^{\circ}$, and taking into account the average beam size $\left(\sim 3^{\prime \prime}\right)$, a limiting radius for an optically thick region for sources at distances of $150 \mathrm{pc}$ is given by

$$
R_{\tau>1}=80[B]^{1 / 2} \mathrm{AU}
$$

where $B$ is the line-center intensity of peak emission (in Jy beam ${ }^{-1}$ ) in a $1.30 \mathrm{~km} \mathrm{~s}^{-1}$ wide velocity bin. This is a conservative upper limit, since contributions from optically thin emission are ignored, and higher temperatures will significantly lower the result. For objects where a sizable fraction of circumstellar mass is still infalling, the average temperature is likely to be higher.

In column (5) of Table $2, R_{\tau>1}$ is listed for each source, taking into account individual beam sizes and subtracting any contribution from the peak continuum flux (never more than $20 \%$ ). A comparison with $R_{\mathrm{HM}}$ reflects the extent to which the emission is optically thin. As will be evident, this is important in estimating the circumstellar mass from the $\mathrm{CO}(2 \rightarrow 1)$ fluxes.

The total mass of circumstellar gas is calculated from the integrated $\mathrm{CO}(2 \rightarrow 1)$ emission, $\int S_{\nu} d v$, using (cf. Scoville et al. 1986):

$$
\begin{aligned}
\mathscr{C}_{\mathrm{H}_{2}}= & 1.42 \times 10^{-10} \frac{\left(T_{x}+0.93\right)}{e^{-16.76} / T_{x}} \frac{\tau_{\mathrm{CO}}}{\left(1-e^{-\tau}\right)} \\
& \times \frac{D_{\mathrm{kpc}}^{2}}{X(\mathrm{CO})} \int S_{\nu} d v \quad \mathscr{C}_{\odot} .
\end{aligned}
$$

Here, $T_{x}$ is the excitation temperature, $\tau_{\mathrm{CO}}$ is the optical depth in the CO line, $D_{\mathrm{kpc}}$ is the distance to the source in $\mathrm{kpc}$, and $X(\mathrm{CO})$ is the fractional abundance of $\mathrm{CO}$. For optically thin emission,

$$
\frac{\tau_{\mathrm{CO}}}{\left(1-e^{-\tau}\right)} \approx 1 \text {, }
$$


TABLE 3. Diagnostics of disk orientation.

\begin{tabular}{ccccc}
\hline \hline (1) & $(2)$ & $(3)$ & $(4)$ & $(5)$ \\
Source & $\Theta_{C O}$ & $\Theta_{\delta V}$ & $\Theta_{p o l}$ & $\Theta_{j e t}+90^{\circ}$ \\
\hline RY Tauri & $48^{\circ} \pm 5^{\circ}$ & $21^{\circ}$ & $20^{\circ} \pm 20^{\circ}{ }_{a}$ & - \\
DL Tauri & $84^{\circ} \pm 5^{\circ}$ & $84^{\circ}$ & $85^{\circ} \pm 7^{\circ}{ }_{b}$ & - \\
DO Tauri & $160^{\circ} \pm 5^{\circ}$ & - & $170^{\circ} \pm 1^{\circ}{ }_{a}$ & $160^{\circ}{ }_{c}$ \\
AS 209 & $138^{\circ} \pm 2^{\circ}$ & $120^{\circ}$ & $134^{\circ} \pm 10^{\circ}{ }_{a}$ & - \\
\hline \hline
\end{tabular}

э) Bastien (1982)

b) Tamura \& Sato (1989)

c) Hirth et al. (1994)

and the mass is calculable without a priori knowledge of $\tau_{\mathrm{CO}}$. If the magnitude of the optical depth is completely unknown, the above approximation allows a determination of a lower limit to $\mathscr{M}_{\mathrm{H}_{2}}$.

Masses, $\mathscr{C}_{\mathrm{H}_{2}}$, were calculated for all the sources using the integrated line fluxes listed in Table 2 , adopting an average value of $T_{x}=30 \mathrm{~K}, X(\mathrm{CO})=10^{-4}, D_{\mathrm{kpc}}=0.140 \mathrm{kpc}$ for the Taurus sources, and $0.160 \mathrm{kpc}$ for AS 209. The results are shown in column (6) of Table 2. As optical depth decreases, $\mathscr{O}_{\mathrm{H}_{2}}$ approaches the true mass; $R_{\tau>1} / R_{\mathrm{HM}}$ provides a qualitative assessment of the accuracy of the estimate. Table 2 shows that, for the entire sample, the upper limit to the radius of an optically thick region equals or exceeds the size of the present solar system (50 AU). Total masses much larger than the limits in Table 2, and still confined within a solar-systemsized radius, could easily be consistent with these observations. Indeed, masses typically 2 orders of magnitude larger are implied by $1.3 \mathrm{~mm}$ continuum measurements (Beckwith et al. 1990; André \& Montmerle 1994). Further observations in optically thin isotopes of $\mathrm{CO}$ are needed to refine estimates of the mass of circumstellar gas.

\subsection{Orientation and Kinematic Implications}

The true orientation of the elongation axis of the circumstellar gas is critical to any kinematic analysis. In Figs. 1(a)4(a), emission is preferentially elongated along one axis, implying a flattened structure, but the degree of elongation is uncertain, since some objects are very small compared to the spatial resolution of the maps. However, the orientation of an associated optical jet can also be used to infer the P.A. of the sky-projected axis normal to the disk plane.

In Table 3 , the P.A. of $\mathrm{CO}$ emission elongation, $\Theta_{\mathrm{CO}}$, determined from the Gaussian fit, is listed for each of the sources. Uncertainties tabulated for $\Theta_{\mathrm{CO}}$ are derived from the goodness of fit and do not reflect the amplitude and position uncertainties in the map itself. Errors for RY Tau, where the deconvolved size is less than the FWHM size of the synthesized beam, are likely to be greater than the formal uncertainties listed in Table 3. In addition, irregular source features can confuse the determination of elongation orientation, especially when spatial resolution is limited. These features include azimuthal asymmetries in the circumstellar gas itself, molecular outflows, or ambient cloud material. Independent ways of constraining the orientation of the circumstellar material must be employed. In fact, the spatial distribution of the intensity-weighted average velocity ("first moment" with respect to velocity) can provide circumstantial evidence about disk orientation, if systematic velocity gradients are apparent. Maps of the first moment in velocity for the sample sources are shown in Figs. 1(b)-4(b).

To a first approximation, rotation in a flattened disk will appear as a gradient in mean velocity parallel to the elongation axis of the disk. Outflow, or pure infall in the plane of a flattened envelope, on the other hand, will result in a gradient orthogonal to this direction. Complex mean velocity structure will result from a combination of these effects, and the velocity gradient alone is not an unambiguous indicator of disk orientation, especially if the elongation itself is due to outflow. However, the molecular gas in maps shown here exhibits much smaller linewidths than typically observed for outflow sources (except for DO Tau) and is positioned close to the star. Therefore, for purposes of preliminary interpretation, the coincidence of a mean velocity gradient with the apparent $\mathrm{CO}$ elongation axis is considered as a significant clue to disk orientation. More detailed kinematic modeling like that carried out for GM Aur by KSB will be presented in a future paper. The P.A. of any systematic velocity gradient seen in Figs. 1(b)-4(b), $\Theta_{\delta V}$, is given in column (3) of Table 3 for comparison with $\Theta_{\mathrm{CO}}$. For RY Tau and AS 209, $\Theta_{\delta V}$ was measured by taking the perpendicular to conteurs at the position of peak emission in Figs. 1(a) and 2(a), since curvature was evident in the velocity gradient.

Other constraints on the orientation of a possible disk include the observation of an optical jet, presumably oriented perpendicular to the axis of elongation. The implied disk orientation is given as $\Theta_{\text {jet }}$ in Table 3. Although $\Theta_{\text {jet }}$ cannot be used alone to define orientation, agreement between several indicators strongly suggests the correct orientation of planar material. The P.A. of spatially averaged polarization vectors of scattered stellar light, $\Theta_{\text {pol }}$, is also a function of the orientation of the scattering material (Elsässer \& Staude 1978; Bastien 1992). Monte Carlo models of light scattered by disks and envelopes indicate that $\Theta_{\text {pol }}$ will be parallel to the elongation axis for flared disks and envelopes, but perpendicular to it for thin disks (Whitney \& Hartmann 1992, 1993). In Table 3, the agreement between $\Theta_{\mathrm{CO}}, \Theta_{\delta v}$, and $\Theta_{\mathrm{pol}}$ is generally quite good and is supported by $\Theta_{\mathrm{jet}}$ in the case of DO Tau (Hirth et al. 1994). We claim this greatly strengthens an interpretation of the structures in Figs. 1-4 as thick disks or flattened envelopes. Thin disks are ruled out, since $\Theta_{\mathrm{pol}}$ would be expected to differ from $\Theta_{\mathrm{CO}}$ and $\Theta_{\delta v}$ by $90^{\circ}$.

\subsection{Velocity Structure of Circumstellar Gas}

Maps of the first moment with respect to velocity for RY Tau, DL Tau, DO Tau, and AS 209, in Figs. 1(b)-4(b), display velocity gradients parallel to the elongation axis of $\mathrm{CO}$ emission and strongly suggest the existence of rotating disks. This evidence is not sufficient to uniquely establish the kine- 


\section{$\mathrm{CO}(2 \rightarrow 1)$ Spectra from T Tauri Stars}
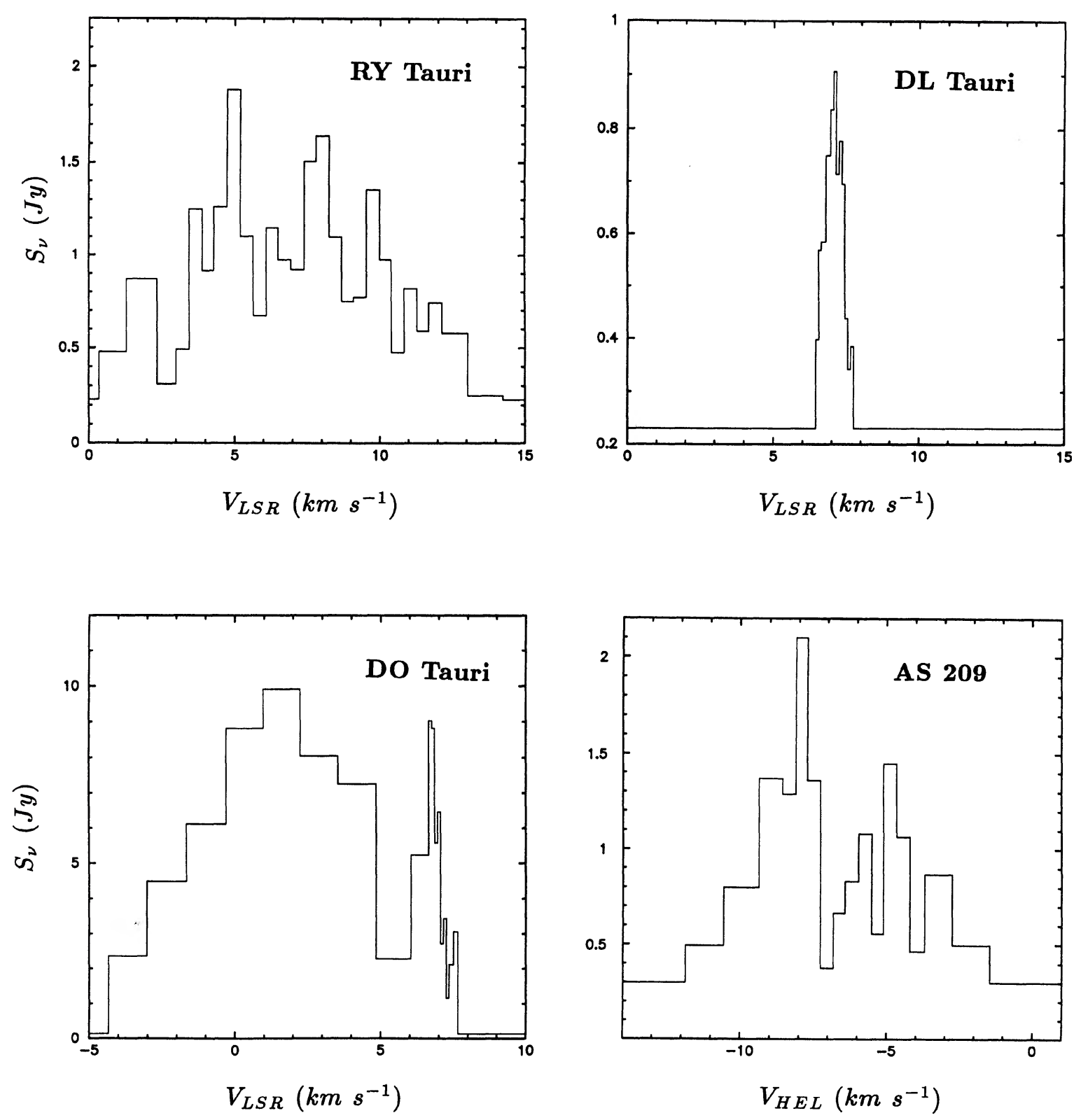

FIG. 5. CO $(2 \rightarrow 1)$ spectra from the T Tauri stars, RY Tau, DL Tau, DO Tau, and AS 209. The integrated intensity from circumstellar emission in high-spatialresolution spectral line maps plotted as a function of $V_{\mathrm{LSR}}\left(V_{\mathrm{HEL}}\right.$ for AS 209). Variations in velocity resolution are the result of limited bandwidth coverage for the high velocity resolution channels. Molecular line shapes are uniformly plotted over a width of $15 \mathrm{~km} \mathrm{~s}^{-1}$ for easy comparison. The continuum flux level, evident as a perfectly flat line for DL Tau, is shown outside the region of spectral coverage. The flux scale, in Jy, is not uniform between spectra, but ranges over an order of magnitude to facilitate comparison of lineshapes.

matic structure of the gas, however, since detailed information about the velocity distribution of emission has been lost by averaging. In Fig. 5 , spatially integrated $\mathrm{CO}(2 \rightarrow 1)$ spectra provide a complementary presentation of the data which retains velocity information. The emission intensity in spectral line maps was integrated over a 5 " radius region centered on the stellar position and plotted as a function of velocity to effectively separate the signature of circumstellar gas from that of the large ambient cloud.

The spectra in Fig. 5 may be used to refine preliminary interpretations of the circumstellar $\mathrm{CO}$ emission. Two of the sources, RY Tau and AS 209, have line shapes which are 
approximately double peaked, consistent with Keplerian rotation (Beckwith \& Sargent 1993b). This interpretation is somewhat uncertain for RY Tau, since the peak-to-trough amplitude is only a few times that of the greatest channel-tochannel variations due to noise. However, it is bolstered by available observations of the stellar radial velocity; the molecular spectrum is centered exactly on the optical value given by Hartmann et al. (1986), $V_{\mathrm{LSR}}=7.8 \pm 2.4 \mathrm{~km} \mathrm{~s}^{-1}$. The line shape for AS 209 is double peaked about a central velocity $V_{\mathrm{HEL}} \approx-7 \mathrm{~km} \mathrm{~s}^{-1}$, consistent with a stellar velocity of $V_{\mathrm{HEL}}=-6 \pm 3 \mathrm{~km} \mathrm{~s}^{-1}$ (Walter 1994). The spectral line for DL Tau is centered at $V_{\mathrm{LSR}} \approx 7 \mathrm{~km} \mathrm{~s}^{-1}$, a typical velocity for $\mathrm{T}$ Tauri stars in the Taurus-Auriga molecular dark cloud (cf. Hartmann et al. 1986). The linewidth is surprisingly narrow $\left(\Delta V=1.2 \mathrm{~km} \mathrm{~s}^{-1}\right)$ such that any kinematic signature is likely to be masked by thermal broadening.

The remaining source, DO Tau, shows an extremely asymmetric spectral line that is an order of magnitude stronger than the others. Most of the molecular emission is blueshifted more than $10 \mathrm{~km} \mathrm{~s}^{-1}$ relative to the velocity of the ambient molecular cloud and the best available stellar velocity $\left(V_{\mathrm{LSR}}=10 \pm 5 \mathrm{~km} \mathrm{~s}^{-1}\right.$; Herbig 1977). The dominant blueshifted wing is most likely due to a molecular outflow, especially since a blueshifted optical jet is known to emanate from the central star (Hirth et al. 1994). Redward of the peak emission, however, there is a narrow feature separated from the blueshifted emission by a prominent dip at the systematic velocity of the local molecular cloud. These features may be circumstellar in origin.

A “double-peaked" line shape arises from a centrifugally supported disk as a result of the $1 / \sqrt{r}$ dependence of the Keplerian velocity, the $r^{2}$ dependence of emitting areas with the same velocity, and the truncation of the disk at some outer radius. Peaks in the spectra largely reflect the Keplerian velocities at the outer radius of the disk, modulated by $\sin (i)$, where $i$ is the angle of inclination to the line of sight. However, other spatial and kinematic configurations can yield a similar lineshape. Line-center absorption by a cold intervening molecular cloud can introduce a central dip in the line shape, especially in ${ }^{12} \mathrm{CO}$ observations where the emission is likely to be optically thick, and infall in a flattened structure also produces a double-peaked line shape, since infall velocities are also proportional to $1 / \sqrt{r}$.

Although none of the spectra in Fig. 5 can be taken as unambiguous proof of the presence of a Keplerian disk, they do provide a consistency check. The Keplerian velocity at the outer radius, $\sqrt{G M / R_{d}}$, can be calculated assuming the central mass is approximately the stellar mass, $\mathscr{l} \approx \approx \mathscr{M}_{*}$, listed in Table 1 , and that the outer disk radius is given by $R_{d} \approx R_{\mathrm{HM}}$ of Table 2 . The derived Keplerian velocity can then be modulated over a range of acceptable inclination angles and compared with double-peak velocities suggested by Fig. 5 . If the center-to-peak velocity difference is significantly greater than that predicted for an edge-on Keplerian disk of the observed size, Keplerian rotation can be ruled out as the dominant cause of the spectral line shape.

Table 4 lists Keplerian velocities calculated in this way and center-to-peak velocity differences estimated from the spectra in Fig. 5. For RY Tau and AS 209, this was simply
TABLE 4. Derived Keplerian disk parameters.

\begin{tabular}{cccc}
\hline \hline (1) & $(2)$ & $(3)$ & $(4)$ \\
Source & $\Delta \mathrm{V}_{\text {spectra }}$ & $\Delta \mathrm{V}_{\text {Kepler }}$ & $i$ \\
& $(\mathrm{~km} / \mathrm{s})$ & $(\mathrm{km} / \mathrm{s})$ & (degrees) \\
\hline RY Tauri & 1.5 & 3.7 & 25 \\
DL Tauri & 0.3 & 1.4 & 12 \\
DO Tauri & 1.0 & 1.4 & 45 \\
AS 209 & 1.5 & 1.8 & 56 \\
\hline \hline
\end{tabular}

taken to be half the distance between the channels with the highest amplitudes. For DL Tau, where the narrowness of the line obscures any double-peaked structure, a nominal value of one-quarter of the total linewidth was chosen. In the case of DO Tau, the distance between the spectral dip and the narrow velocity feature was used. None of the velocities estimated from the spectra exceed those of the Keplerian approximation. In face, since free fall velocities exceed the Keplerian velocity by a factor of $\sqrt{2}$, this is more consistent with a rotational rather than an infall interpretation. The range of inclination angles required for agreement with Keplerian rotation is given in column 4 . The values are plausible and, in the case of the best-resolved disk, consistent with an estimate based on the aspect ratio of the major and minor axes of $\mathrm{CO}$ emission.

\subsection{Combined Evidence for Rotating Disks around Individual Stars}

None of the independent diagnostics presented thus far demonstrate conclusively that the objects observed are protoplanetary disks. However, their combined effect provides useful criteria with which to test the simplest possible interpretations. These include a rotating disk, infall envelope, bipolar outflow, or some combination of these; more complicated explanations can perhaps be constructed, but their consideration is unwarranted unless these hypotheses are ruled out. With these restrictions in mind, it is useful to briefly summarize the diagnostics for each individual star in order to assess the likelihood that observed CO emission arises from a protoplanetary disk.

RY Tau. Circumstellar CO emission around RY Tau is compact, located at the stellar position, and exhibits a symmetric double-peaked line shape centered at the radial stellar velocity. The linewidth is consistent with that from a disk inclined $25^{\circ}$ from face on with radius $\sim 100 \mathrm{AU}$ and in Keplerian rotation about a central mass dominated by that of the star. Indicators of orientation-the P.A.'s of CO elongation, velocity gradient, and polarization-also support a rotating-disk interpretation and are aligned within $20^{\circ}$ of each other. The observed values are somewhat uncertain due to the small radial extent of $\mathrm{CO}$ and variability of polarization, but their validity is confirmed by the orientation of a reflection nebulosity associated with RY Tau in $R$ - and $I$-band coronagraphic images (Nakajima \& Golimowski 
1995). Further, the compact size, line shape, and linewidth argue that the $\mathrm{CO}$ is truly circumstellar and not part of an outflow. It must be conceded, however, that an element of infall is still possible, and a compact low-velocity outflow is not strictly excluded.

DL Tau. Orientation indicators for circumstellar gas around DL Tau are in exact agreement and strongly support the idea that $\mathrm{CO}$ emission represents a $250 \mathrm{AU}$ radius circumstellar disk. The spectral line is centered at a typical velocity for $\mathrm{T}$ Tauri stars in the Taurus-Auriga molecular dark cloud and is surprisingly narrow. A nearly face-on disk orientation is required to match it, given the stellar mass estimate of $0.56 \mathscr{C}_{\odot}$. However, pre-main-sequence stellar mass estimates are most uncertain for stars of very low mass (cf. d'Antona \& Mazzitelli 1993), and DL Tau is the least massive of the sample. It is also possible that the observed linewidth has been partially narrowed by absorption from the ambient molecular cloud. In either case, the narrow linewidth and compact morphology argue against an outflow interpretation and the orientation diagnostics overwhelmingly support a disklike configuration. If this is truly the case, infall is strictly excluded by the orientation of the velocity gradient with respect to that of the elongation axis. In these respects, the rotating disk interpretation is robust for DL Tau. To confirm this, imaging at higher spatial resolution is needed to resolve velocity-dependent structure for comparison to a kinematic model.

DO Tau. The orientation of circumstellar gas around DO Tau is well established by the morphology of $\mathrm{CO}$ emission and observations of its optical jet and polarization. However, a single systematic velocity gradient is not apparent in the first moment velocity map. The line shape is much broader than expected for rotation and asymmetrically blueshifted to velocities higher than would be expected for Keplerian rotation in a disk that size. Its association with an asymmetric optical jet virtually establishes an outflow origin for the highvelocity molecular emission. In contrast, a disklike appearance and agreement between three orientation indicators argue for a large circumstellar component of rotating and/or infalling gas. Spectral features near the systemic velocity are consistent with Keplerian rotation, but detailed study of the emission morphology at these velocities is required to identify any rotating disk. Clearly, some combination of hypotheses is needed to explain the morphology and kinematics of DO Tau's CO emission. To obtain the correct interpretation, modeling of emission in individual spectral line maps is being carried out and will be presented in a future paper.

$A S$ 209. The map of $\mathrm{CO}$ emission reveals an elliptical shape with somewhat tenuous extensions perpendicular to the elongation axis. The dominant velocity gradient and polarization vector are parallel to this axis, but the velocity gradient is not as clear as for DL Tau and RY Tau. The line shape is double peaked about a central velocity that agrees approximately with estimates of the stellar velocity, and the linewidth is consistent with a Keplerian disk inclined $\sim 25^{\circ}$ from edge on. Taken together, these facts suggest that the emission is dominated by a rotating disk. However, the perpendicular extensions, irregular velocity gradient, and a slight asymmetry in the line shape could easily be accounted for by an additional outflow component to the molecular emission. As for DO Tau, modeling of high spatial- and velocity-resolution spectral line maps is required to confirm this picture and will be the subject of a future paper.

\section{SUMMARY AND DISCUSSION}

Four T Tauri stars, RY Tau, DL Tau, DO Tau, and AS 209, have been imaged in the $\mathrm{CO}(2 \rightarrow 1)$ line at $\sim 2^{\prime \prime}-3^{\prime \prime}$ resolution. In all cases, emission is resolved with deconvolved half-maximum radii of 100-350 AU. The P.A.'s of optical linear polarization and, in one case, an optical jet show remarkable agreement with disk orientation implied by the $\mathrm{CO}$ elongation and by velocity gradients. From the peak strength of line emission, upper limits on the size of any optically thick inner region are found to be 50-150 AU, the approximate size of the solar system. Masses of circumstellar $\mathrm{H}_{2}$ range over 2 orders of magnitude, from $1.3 \times 10^{-6}$ to $1.4 \times 10^{-4} \mathscr{l}_{\odot}$, but these values are lower limits and much less than masses estimated from the dust continuum emission (Beckwith et al. 1990; André \& Montmerle 1994). Except for DO Tau, all sources show evidence of a velocity gradient along the elongation axis and spectral line shapes and linewidths which are consistent with Keplerian rotation about the estimated stellar mass.

The strongest case for a centrifugally supported disk can be made for circumstellar gas around DL Tau. With RY Tau, it shows the most distinct velocity gradient in first moment velocity maps. For both AS 209 and RY Tau, however, the uncertainty in orientation admits a possible infall or outflow component to circumstellar gas. Nevertheless, the P.A. of elongation is not likely to be off by as much as $45^{\circ}$, indicating that the dominant velocity component of the molecular gas is rotational. Spectral line shapes for all three objects are also consistent with that of a Keplerian disk and strengthen the case for a rotational interpretation of the velocity gradients observed in moment maps. If the possible kinematic interpretations are limited to rotation, outflow, or infall, evidence presented here weighs heavily in favor of predominantly rotation of circumstellar gas for all three of these sources.

For DO Tau, the first moment velocity map and associated spectrum eliminates the possibility that all of the observed emission radiates from rotating circumstellar gas. An outflow interpretation for some of the gas is supported by the presence of an optical jet (Hirth et al. 1994). It is somewhat surprising, therefore, that the morphology of molecular emission in Fig. 3 resembles a disk with orientation perpendicular to the jet. The disklike morphology and complex velocity structure may indicate the presence of a radially infalling component to the circumstellar velocity structure [e.g., Hayashi et al. 1993; for theoretical justification of a flattened infall region, see Galli \& Shu (1993) and Stahler et al. (1994)]. Molecular material around DO Tau, with $R_{\mathrm{HM}}=350$ $A U$, is substantially more extended than that around the other sources, but is still more compact than for many earlier objects such as HL Tau. It may represent an intermediate evolutionary state in which much of the circumstellar material is centrifugally supported, but some is still in a state of 
infall. Modeling of high-spatial-resolution spectral line maps of DO Tau with a kinematic model of infall and rotation is required to identify such a kinematic state (Koerner 1994) and will be presented in a future paper.

In the above respects, circumstellar $\mathrm{CO}(2 \rightarrow 1)$ emission from the $\mathrm{T}$ Tauri stars surveyed shows characteristics which confirm the hypothesis that these stars are surrounded by protoplanetary disks like that which gave rise to our solar system. Further work is needed to secure this interpretation. Kinematic modeling of spectral line maps of ${ }^{13} \mathrm{CO}(2 \rightarrow 1)$ emission from GM Aurigae provided satisfactory evidence for Keplerian rotation of circumstellar gas by duplicating systematic patterns of emission seen in maps of the disk at discrete velocities (KSB). However, the circumstellar gas structures described here are much smaller and similar analyses will require imaging at correspondingly higher spatial resolution.

Despite the inability to rigorously identify kinematic patterns, this work confirms the presence of molecular gas in the circumstellar environment of $\mathrm{T}$ Tauri stars for which dust continuum emission is detected at millimeter wavelengths. The radial extent of the gas is more in accord with the dimensions of the solar system than that of previously imaged T Tauri stars; molecular line emission is resolved in $2^{\prime \prime}-3^{\prime \prime}$ FWHM beams (corresponding to 300-450 AU) and optically thick beyond a radial distance corresponding to the semimajor axis of Pluto's orbit ( $\sim 50 \mathrm{AU})$. Some evidence of centrifugal support is present for most of the sample. These facts strongly support that hypothesis that a large fraction of $\mathrm{T}$ Tauri stars are surrounded by protoplanetary disks similar to the early solar nebula.

We thank the anonymous referees for comments which helped clarify the results of this paper. D.K. acknowledges support from NASA Grant No. NGT-51071 and a Resident Research Associateship from the National Research Council. Owens Valley Radio Observatory is funded by NSF Grant No. AST-9314079.

\section{REFERENCES}

André, P., \& Montmerle, T. 1994, ApJ, 420, 837

Bastien, P. 1982, A\&AS, 48, 153

Beckwith, S. V. W., \& Sargent, A. I. 1991, ApJ, 381, 250

Beckwith, S. V. W., \& Sargent, A. I. 1993a, in Protostars and Planets III, edited by E. H. Levy and J. L. Lunine (University of Arizona Press, Tucson), p. 521

Beckwith, S. V. W., \& Sargent, A. I. 1993b, ApJ, 402, 280

Beckwith, S. V. W., Sargent, A. I., Chini, R., \& Güsten, R. 1990, AJ, 99, 924

Beckwith, S. V. W., Sargent, A. I., Scoville, N. Z., Masson, C. R., Zuckerman, B., \& Phillips, T. G. 1986, ApJ, 309, 755

Cohen, M., Emerson, J. P., \& Beichman, C. A. 1989, ApJ, 339, 455

Cohen, M., \& Kuhi, L. V. 1979, ApJS, 41, 743

d'Antona, F., \& Mazzitelli, I. 1993, ApJS, 90, 467

de Geus, E. J., Bronfman, L., \& Thaddeus, P. 1990, A\&A, 231, 137

Dutrey, A., Guilloteau, S., \& Simon, M. 1994, A\&A, 286, 149

Elias, J. 1978, ApJ, 224, 857

Elsasser, H., \& Staude, H. J. 1978, A\&A, 70, L3

Galli, D., \& Shu, F. H. 1993, ApJ, 417, 220

Ghez, A., Neugebauer, G., \& Mathews, K. 1993, AJ, 106, 2005

Hartmann, L., Hewett, R., Stahler, S., \& Mathieu, R. D. 1986, ApJ, 309, 275

Hayashi, M., Ohashi, N., \& Miyama, S. M. 1993, ApJ, 418, L71

Henning, T., Pfau, W., Zinnecker, H., \& Prusti, T. 1994, A\&A, 276, 129

Herbig, G. H. 1977, ApJ, 214, 747

Herbig, G. H., \& Bell, K. R. 1988, in Lick Obse. Bull. No. 1111 (University of California)

Hirth, G. A., Mundt, R., Solf, J., \& Ray, T. P. 1994, ApJ, 427, L99

Kawabe, R., Ishiguro, M., Omodaka, T., Kitamura, Y., \& Miyama, S. M. 1993, ApJ, L63

Kenyon, S. J., Calvet, N., \& Hartmann, L. 1993, ApJ, 414, 676

Koerner, D. W. 1994, Ph.D. dissertation, California Institute of Technology
Koerner, D. W., Sargent, A. I., \& Beckwith, S. V. W. 1993a, Icarus 106, 2 Koerner, D. W., Sargent, A. I., \& Beckwith, S. V. W. 1993b, ApJ, 408, L93 Leinert, Ch., Hass, M., Richi, A., Zinnecker, H., \& Mundt, R. 1991, A\&A, 250, 407

Nakajima, T., \& Golimowski, D. A. 1995, AJ (in press)

Ohashi, N., Kawabe, R., Hayashi, M., \& Ishiguro, M. 1991, AJ, 102, 2054

Sargent, A. I., \& Beckwith, S. 1987, ApJ, 323, 294

Sargent, A. I., \& Beckwith, S. V. W. 1989, in Structure and Dynamics of the Interstellar Medium, IAU Colloquium No. 120, p. 215

Sargent, A. I., \& Beckwith, S. 1991, ApJ, 382, L31

Sargent, A. I., \& Beckwith, S. 1993, in Millimeter and Submillimeter Wave Interferometry, edited by M. Ishiguro and R. Kawabe (Bookcrafters, San Francisco), p. 232

Scoville, N. Z., Sargent, A. I., Sanders, D. B., Claussen, M. J, Masson, C. R., Lo, K., \& Phillips, T. G. 1986, ApJ, 303, 416

Stahler, S. W., Korycansky, D. G., Brothers, M. J., \& Touma, J. 1994, ApJ, 431, 341

Stapelfeldt, K. R. et al., 1995, ApJ (in press)

Strom, K. M., Strom, S. E., Edwards, S., Cabrit, S., \& Skrutskie, M. F. 1989, AJ, 97, 1451

Tamura, M., \& Sato, S. 1989, AJ, 98, 1368

Ungerechts, H., \& Thaddeus, P. 1987, ApJS, 377, 510

van Langevelde, H. J., van Dishoeck, E. F., \& Blake, G. A. 1994, ApJ (in press)

Walter, F. M., 1994, private communication

Weaver, W. B., \& Jones, G. 1992, ApJS, 78, 239

Weintraub, D. A., Masson, C. R., \& Zuckerman, B. 1987, ApJ, 320, 336

Weintraub, D. A., Masson, C. R., \& Zuckerman, B. 1989, ApJ, 344, 915

Whitney, B. A., \& Hartmann, L. 1992, ApJ, 395, 529

Whitney, B. A., \& Hartmann, L. 1993, ApJ, 402, 605

Whittet, D. C. B. 1974, MNRAS, 168, 371 